\title{
Absenkung beim Weiterbildungstitel «Praktischer Arzt / Praktische Arztin»
}

\section{Patrick Müller}

Leiter Abteilung Ambulante Versorgung und Tarife

\author{
Der Bundesrat verordnete per 1. Januar 2018 einen zweiten Tarifeingriff mit um- \\ fangreichen Massnahmen in den TARMED. Eine dieser vom Bundesrat verordneten \\ Massnahmen betrifft die Einführung eines einheitlichen Dignitätsfaktors und den \\ Skalierungsfaktor beim Weiterbildungstitel «Praktischer Arzt / Praktische Ärztin». \\ Die Anwendung dieser verordneten Absenkung führte zu vielen Rückfragen.
}

Nach dem Scheitern der tarifpartnerschaftlichen Revision im Sommer 2016 führte der Bundesrat mit seiner subsidiären Kompetenz einen zweiten und sehr umfangreichen Tarifeingriff in den TARMED per 1.1.2018 durch. Über die einzelnen Details dieser Massnahmen wurde in der Schweizerischen Ärztezeitung bereits berichtet [1]. In einer Massnahme führte der Bundesrat einen einheitlichen Dignitätsfaktor von 0.985 ein und ebnete damit die «Quantitativen Dignitäten" ein. Damit wird der Minutenkostensatz der Ärztlichen Leistung (AL) unabhängig von der ärztlichen Weiterbildung und Tarifposition festgelegt. Eine Ausnahme machte er beim Weiterbildungstitel «Praktischer Arzt / Praktische Ärztin»: hier verordnete der Bundesrat, dass Ärztinnen und Ärzte, welche ausschliesslich über einen solchen Weiterbildungstitel und nicht über einen Facharzttitel verfügen, einen Skalierungsfaktor (Reduktion) von 0.93 auf die AL anwenden müssen (Basis sind die neuen AL Taxpunkte der Tarifpositionen gemäss TARMED-Version 1.09_BR). Das BAG, welche die Verordnung tarifarisch umsetzte, hat es bisher unterlassen, eine genaue Definition der Anwendung dieses Skalierungsfaktors zu publizieren. Damit verbunden erreichten die FMH in den letzten Wochen sehr viele Rückfragen, was nun genau unter «Praktischer Arzt / Praktische Ärztin» verstanden und wie dieser Skalierungsfaktor angewendet wird.

\section{Weiterbildungstitel «Praktischer Arzt / Praktische Ärztin»}

Die Schweiz hat den eidgenössischen Weiterbildungstitel «Praktischer Arzt / Praktische Ärztin» im Zuge der Bilateralen Verträge mit der EU geschaffen und im Jahr
2002 in Kraft gesetzt. Im Anschluss an ein 6-jähriges Medizinstudium erlaubt dieser lediglich drei Weiterbildungsjahre und eine Schlussprüfung umfassende Minimaltitel den Erwerb einer kantonalen Berufsausübungsbewilligung und damit die Aufnahme einer selbständigen ärztlichen Tätigkeit in der ganzen Schweiz. Wer über einen in einem Mitgliedstaat der EU erworbenen Weiterbildungstitel "Allgemeinmedizin» verfügt, der in der EU-Richtlinie 2005/36 unter Ziff. 5.1.4. "Ausbildungsnachweise für den Allgemeinmediziner» aufgeführt ist, kann aufgrund des Freizügigkeitsabkommens Schweiz/EU in der Schweiz nur als «Praktischer Arzt / Praktische Ärztin» anerkannt werden. Dabei wird im Rahmen der Anerkennung von ausländischen Allgemeinmedizin-Weiterbildungstiteln durch die MEBEKO nicht berücksichtigt, ob es sich beim ausländischen Weiterbildungstitel um eine 5-jährige oder eine 3-jährige Qualifikation handelt. Beim Titel «Praktischer Arzt / Praktische Ärztin» handelt es ausdrücklich nicht um einen Facharzttitel, was u.a. zur Folge hat, dass er z.B. keine genügende Grundlage für den Erwerb von Fähigkeitsausweisen bildet.

Der Weiterbildungstitel «Praktischer Arzt / Praktische Ärztin» darf nicht mit der Bezeichnung «med. pract.» verwechselt werden. Früher haben sich Ärztinnen und Ärzte, die über keinen Doktortitel verfügten, oft als «med. pract.» bezeichnet. Damit wird jedoch nichts über die absolvierte Weiterbildungszeit resp. Facharztweiterbildung ausgesagt - es zeigt ausschliesslich, dass die Ärztin oder der Arzt über keinen Doktortitel verfügt. Heute empfiehlt die FMH wegen der Verwechslungsgefahr mit dem «Praktischen Arzt» diese Bezeichnung nicht mehr zu verwenden und an deren Stelle «dipl. Arzt» zu führen. 


\section{Wann muss der Skalierungsfaktor ange- wendet werden?}

Gemäss Verordnung des Bundesrates müssen Ärztinnen und Ärzte, welche ausschliesslich über den Weiterbildungstitel "Praktischer Arzt / Praktische Ärztin" verfügen, auf jeder erbrachten Tarifposition auf der Ärztlichen Leistung (AL, Taxpunkte) den Skalierungsfaktor in der Höhe von 0.93 anwenden. Damit reduzieren sich die Anzahl Taxpunkte der AL für jede erbrachte Tarifposition. Der Faktor soll transparent auf Tarifpositionsebene auf der Rechnung ausgewiesen werden (im Feld fAL des XML-Rechnungsformulars). Dabei kommt der Skalierungsfaktor auch bei Tarifpositionen zur Anwendung, die im Besitzstand der Ärztin / des Arztes sind. Ärztinnen und Ärzte, welche zusätzlich zum Weiterbildungstitel «Praktischer Arzt / Praktische Ärztin" einen eidgenössischen oder in der Schweiz formell anerkannten Facharzttitel erworben haben (Doppeltitelträger), sind von dieser Absenkung nicht betroffen und müssen den Skalierungsfaktor bei keiner Tarifposition (auch nicht bei der Erbringung von Leistungen der Grundversorgung) anwenden.

Werden beispielsweise auf einer Rechnung Leistungen von einem Praktischen Arzt und einem Facharzt aufgeführt, müssen nur die Leistungen des Praktischen Arztes um den Skalierungsfaktor reduziert werden. Dies gilt auch für Rechnungen, die unter einer gemein-
Was tun, falls eine Rechnung wegen fehlendem Skalierungsfaktor zurückgewiesen wird?

Wir haben Hinweise dafür, dass die Versicherungen die richtige Anwendung des Skalierungsfaktors genau prüfen. Falls eine Rechnung durch eine Versicherung mit der Begründung, der Skalierungsfaktor sei nicht angewendet worden, zurückgewiesen wird, bestehen folgende Möglichkeiten:

- Überprüfung der erfassten Angaben des betroffenen Arztes bzw. der betroffenen Ärztin im Medizinalberuferegister unter www.medregom.admin.ch

- Ist in diesem Register ein Facharzttitel aufgeführt, erfolgte die Rückweisung zu Unrecht. In solchen Fällen sollten Sie sich an die SASIS AG wenden, welche für die Versicherungen die Listen der Weiterbildungstitel führt, oder Sie klären den Sachverhalt direkt mit der Versicherung.

- Bei weiteren Fragen (u.a. wenn kein Weiterbildungstitel eingetragen ist) können Sie sich an die Abteilung Ambulante Versorgung und Tarife unter tarife.ambulant[at]fmh.ch wenden.

samen ZSR-Nummer (z.B. bei einer Institution oder Gemeinschaftspraxis) verrechnet werden - Ausschlaggebend ist immer der ausführende Arzt auf Tarifpositionsebene.

Literatur

1 Schweiz Ärztezeitung. 2017;98(47):1568-71. Schweiz Ärztezeitung. 2017;98(28-29):888-91. 